\begin{tabular}{ccc}
\hline International Journal of Engineering \& Technology, $7(3.15)(2018) 321-323$ \\
SPC & Website w ww.sciencepubco.com/index.php/IJET \\
Research paper & Technology \\
\hline
\end{tabular}

\title{
Clusters in the Gas Dynamics and Mathematical Modeling in Mathcade the Results of the Study
}

\author{
Akdana Tashimbetova, Ayman Rysbaeva, \\ Zhazira Suleimenov, Zhanar Kalybekova, Damelkan Sydykova
}

International educational Corporation, Kazakh Academy of transport and communication, Kazakhstan, Almaty

\begin{abstract}
The concentration of clusters and the compressibility factor of nitrogen and oxygen under various conditions are calculated on the basis of the cluster model of dense gas and the Van der Waals equation. As practice shows, the cluster model gives the best agreement with experiment.
\end{abstract}

Keywords: the cluster, baric dependences of the viscosity of dense gases, subcomponents

At present, a large number of equations for the state of real gases is known ${ }^{[1-3]}$. Typically, the non-ideal of a gas is taken into account by amending the ideal gas equation. It is assumed that the number of moles is not dependent on the macro parameters, which is only true in the case when the gas does not form a polymolecular formation. The nature of the interactions of molecules as electrodynamic systems, which have huge accelerations at collisions, is complex, so there is a reason to assume that such formations exist ${ }^{[4,5]}$. The recognition of their existence requires the development of the ways to describe them. One of the methods that allows classifying polymolecular formations is based on the cluster model of the gas state.

It is convenient to reflect gas deviations from an ideal equation of state a compressibility factor ${ }^{[3-10]}$ :

$z=\frac{p V_{M}}{R T}$,

where $V_{M}$ - molar volume, $p$ - -pressure, $T$ - temperature, $R$ -universal gas constant.

The known equations of state can lead to the same form and get the formula for the compressibility factor. Particularly, the van der Waals equation gives the following formula for the compressibility factor:

$$
z=\frac{1}{1+\frac{a}{p V_{M}^{2}}-\frac{b}{V_{M}}-\frac{a b}{p V_{M}^{3}}},
$$

where $a$ and $b$ - van der Waals amendments.

Not to exceed accuracy, the molar volume is used in the calculations, calculated from the ideal gas equation or tabular data: $V_{M}=R T / p$.

$$
V_{M}=R T / p
$$

In the cluster model of a gas, the deviation from the ideal is mainly determined by the change in the number of moles with changes in macro parameters, since the formation of new clusters leads to the disappearance of structural elements of pressure, and their collapse - to the appearance of those. Through the cluster concentrations, the compressibility factor is expressed by the formula:

$$
\mathrm{Z}=\frac{1}{\left(1-b_{c}\right)} \sum_{g=1}^{r} C_{g}^{(n)}
$$

where $b_{c}$ - amendment at its own volume of the molecules.

Due to the huge variety of different orientations of the particles during their collisions, on average, molecules can be considered as balls with effective collision diameter, which gives the following formula for the correction of the eigenvalue:

$$
b_{c}=\frac{2 \psi}{3} n^{(n)} \pi \sigma^{3},
$$

where $\sigma$ - is the effective diameter of the collisions of molecules, $\psi$ - is the packing parameter considering changes of average volume of the molecules entering clusters, $n^{(n)}$ - is the number of molecules per unit volume.

In cluster gas clusters of different sizes are considered as independent components of the mixture in equilibrium with each other and with molecules, so the average speed of the thermal motion of the clusters is inversely proportional to the root of the mass of the cluster. Such mixture can be described by the equation of state with the molar mass determined by the rule of finding the molar mass of the mixture ${ }^{[6,7]}$ :

$$
\langle\mathrm{M}\rangle=\sum_{g=1}^{r} C_{g}^{(c)} \mathrm{M}_{g},
$$

where $C_{g}^{(c)}$ - is the concentration of dimensional clusters relative to the total numerical density of all clusters, 
$\mathrm{M}_{g}$ - - molar mass, $g$--dimensional cluster,

$$
\mathrm{M}_{g}=\mathrm{Mg} \text {. }
$$

The concentration, in contrast, is introduced as the ratio of the partial numerical density of the cluster under consideration to the sum of the numerical densities of all existing clusters:

$$
C_{g}^{(c)} \equiv \frac{n_{g}}{\sum_{g=1}^{r} n_{g}} \equiv \frac{n_{g}}{n^{(c)}},
$$

where $r$ - is the size of the largest cluster that is included in the consideration,

$n_{g}$ - numerical density $g$ - of dimensional clusters,

$n^{(c)}$ - the numerical density of the sum of all clusters.

Using this definition, one can get the formula for calculating the concentration. To do this, the equation of state of the cluster mixture is used:

$$
p=\frac{\rho}{(1-b)\langle\mathrm{M}\rangle} R T \text {, }
$$

where $\rho$ - is the density of the cluster mixture.

To obtain a closed system of equations, from which it is possible to calculate the concentration of the clusters, we express them through the concentration of monomers (molecules) ratio:

$$
C_{1}^{(c)}\left(1+\sum_{g=2}^{r} \exp [-\beta(g-1)]\right)=1,
$$

where $C_{1}^{(c)}$ the proportion of monomers relative to the total number of particles-clusters, $\beta$ - normalization factor.

The equation (9) - allows us to express the concentration through the measured value - through the gas density under the considered conditions:

$$
C_{1}^{(c)} \sum_{g=1}^{r}\{g \exp [-\beta(g-1)]\}=\frac{\rho R T}{p \mathrm{M}_{1}(1-b)} .
$$

The concentration determined in relation to the numerical density of molecules in the formula (4) for the compressibility factor is expressed in the following ratio:

$$
C_{g}^{(n)}=\frac{n_{g}}{n^{(n)}}=\frac{n_{g}}{\sum_{g=1}^{r} g n_{g}}=C_{g}^{(c)} \frac{n^{(c)}}{\sum_{g=1}^{r} g n_{g}}=C_{g}^{(c)} \frac{1}{\sum_{g=1}^{r} g C_{g}^{(c)}}
$$

The above-mentioned formulas show that the concentration of the clusters and the compressibility factor can be calculated if the tabular values of density at a certain pressure and temperature are used $^{[8-10]}$. Calculations for nitrogen and oxygen in such a scheme are given in graphs and tables. The effective diameter of the particle collisions should also be used for calculations. These data are obtained using the effective diameters calculated from the viscosity coefficient at atmospheric pressure ${ }^{[6,7] .}$ It is convenient to use the three-dimensional schedule for searching of interesting areas of macroparameters at which some features come to light. Figure 4 shows the area of the greatest change in the compressibility factor from the pressure. From the above-mentioned data, it can be seen that at high pressures the van der Waals equation gives large deviations from the experiment and from the cluster model, which is due to the significant influence of the change in the number of moles at significant concentrations of clusters. The obtained data suggest that there are clusters of different sizes in gases at high pressures, and their accounting gives a good agreement of the calculated compressibility factor with the experimental data ${ }^{[8,9]}$.

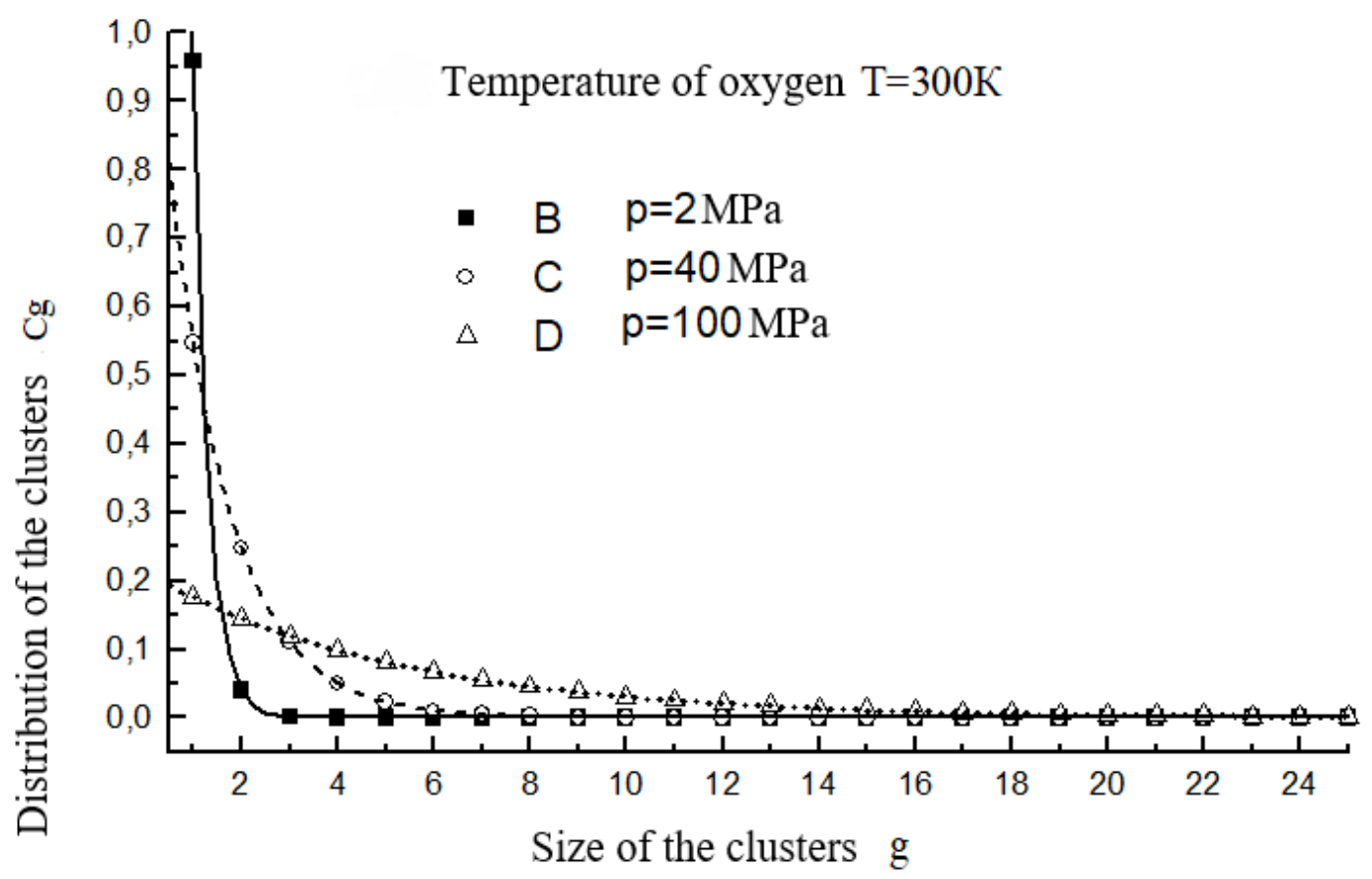

Figure 1:. The distribution of cluster concentrations at the size of the oxygen at a temperature of $300 \mathrm{~K}$ at different pressures.

Line - calculation according to the formula of exponential distri-

bution: 


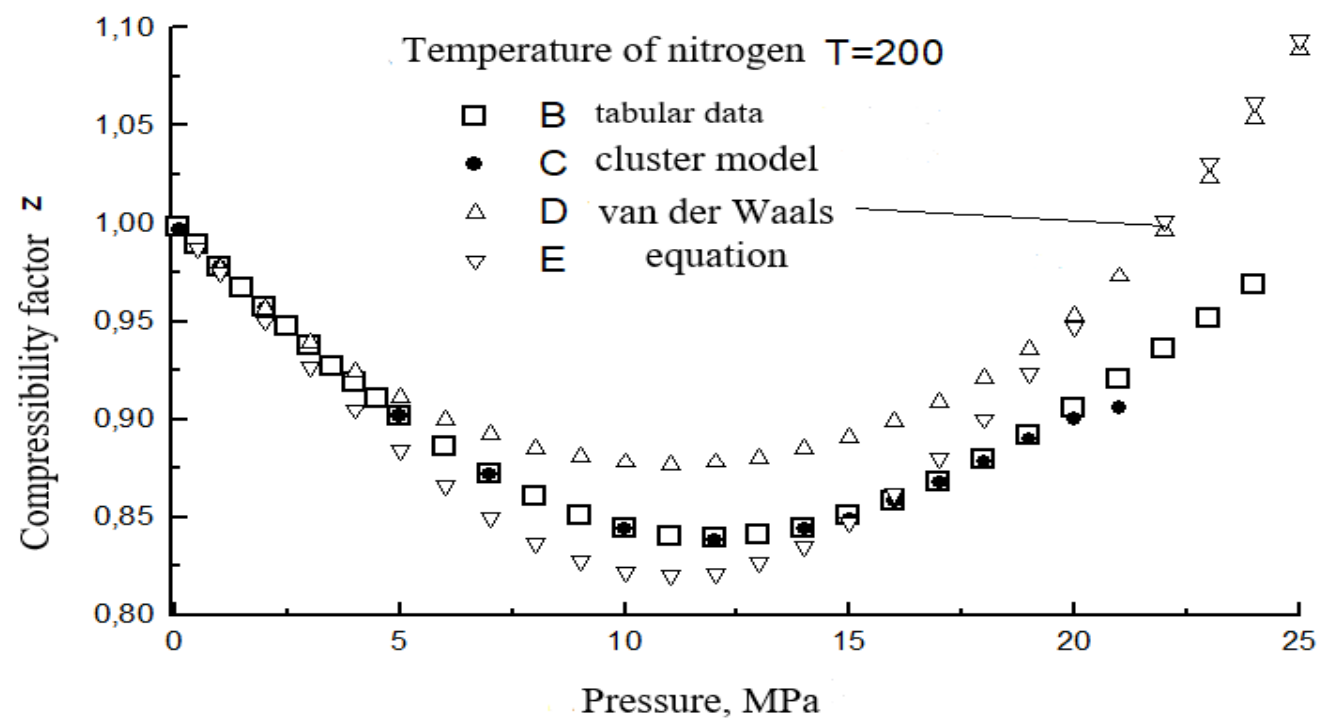

Figure 2:. The pressure dependence of the compressibility factor of nitrogen at a temperature of $200 \mathrm{~K}$.

$\mathrm{V}$ - tabular data ${ }^{[8]}$, the calculation formulas of the cluster model using the effective diameters of the molecules found in the viscosity, D - the calculation of the equation of van der Waals, as amended, and with the ideal-gas molar volume, E - calculation according to the equation of van der Waals tabular molar volume.

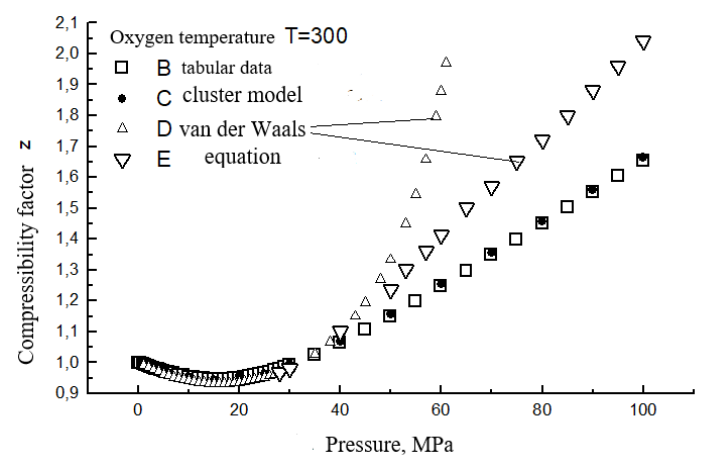

Figure 3:. The pressure dependence of compressibility factor of oxygen at a temperature of $300 \mathrm{~K}$.

V - tabular data ${ }^{[9]}$, the calculation formulas of the cluster model using the effective diameters of the molecules found in the viscosity ${ }^{[6-8]} \mathrm{D}$ - calculation according to the equation of van der Waals, as amended, and with the ideal-gas molar volume, E - calculation according to the equation of van der Waals tabular molar volume.

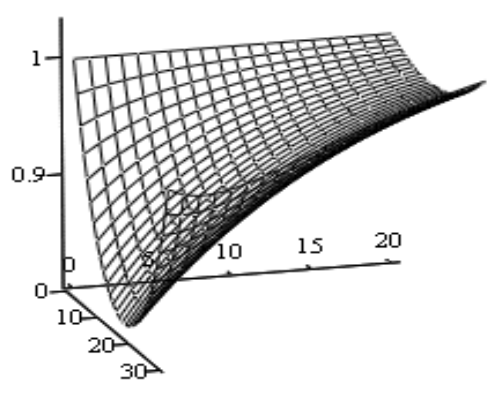

Figure 4:. Oxygen compressibility factor as a function of pressure (on the axis from 0 to $30 \mathrm{MPa}$ ) and temperature (on the axis from 0 to 20 in tens of Kelvin). Calculations made using the van der Waals equation.

\section{References}

[1] Vukalovich M. P. and Novikov I. I. (1948). Equations of state of real gases. Moscow Gosenergoizdat, pp. 340

[2] Hvolson O. D. (1922). physics Course (in five volumes). Volume 1. Ed. 5. R. S. F. S. R. State. ed., Berlin, pp. 676.

[3] Reid, R., J. Prausnitz. and Sherwood T. (1982). Properties of gases and liquids: Handbook. 3rd ed. Leningrad: Chemistry, pp. 592.

[4] Hirschfelder J., Curtiss C. F., bird R. B. (1961). Molecular theory of gases and liquids. Moscow: IL, 1961.-930 S.

[5] Kondratyev V. N. and Nikitin E. E. Kinetics and mechanism of gasphase reactions. Moscow: Science, pp. 558.

[6] Kurlapov L. I. (2000). Kinetic theory of irreversible processes in gases]. Almaty, pp. 300

[7] Kurlapov L. I. Physics of kinetic phenomena in gases. Monograph. Almaty, pp. 271

[8] Sychev V. V. and others (1977). Thermodynamic properties of nitrogen. Moscow: publishing house of standards, pp. 352.

[9] Sychev V. V. (1981). Thermodynamic properties of oxygen: gssd. Series of monograph. Moscow: Publishing house of standards, pp. 304.

[10] Vargaftik N. B. (1972). Handbook of thermophysical properties of gases and liquids. Moscow: Science, pp. 720. 